\title{
The relation between malnutrition screening tool (NUTRIC) score and serum pro- and anti-inflammatory cytokines levels and some nutritional parameters in patients with traumatic head injury in Sulaimani
}

DR SHAHOW ABDULREHMAN EZZADDIN

MBChB, MSc, PhD in Community Medicine, Lecturer in Family and Community Medicine Department, College of Medicine, University of Sulaimani, Sulaimani, Kurdistan Region-Iraq.

Shahow.ezzaddin@univsul.edu.iq

\begin{abstract}
Background: Research has provided evidence that malnutrition screening tool (NUTRIC) score has a relation with interleukin-6 but not with other cytokines. In addition, the relation between malnutrition screening tool (NUTRIC) score and some nutritional parameters has not yet been studied in traumatic head injured patients. Therefore, the aim of the current study is examining the relation between malnutrition screening tool (NUTRIC) score and serum pro- and anti-inflammatory cytokines levels and some nutritional parameters in patients with traumatic head injury in the intensive care unit (ICU) of General Teaching Hospital in Sulaimani, the Kurdistan Region of Iraq in 2016-17.

Methods: Using appropriate methods and tools, the patients' (NUTRIC) score, mid-upper arm circumference (MUAC), disease severity and organ dysfunction, concentration level of serum cytokines (IL-6) were assessed within 24 hours after admission. All of the mentioned measurements except the serum interleukin were also assessed on days 6 and 13 of staying in ICU. Afterwards, in order to examine the relationship between the study's variables, the collected data were analyzed using Mann-Whitney U test through SPSS 22.0.

Results: The results of data analysis using Mann-Whitney $U$ and independent samples T-tests revealed that there was a significant relation between malnutrition screening tool (NUTRIC) score and the studied interleukin (IL-6) within 24 hours after ICU admission. Other studied variables that had a significant association with the patients' (NUTRIC) score within 24 hours after ICU admission were APACHE II score, SOFA score, and MUAC, this significant association has been seen on days 6 and 13 after ICU admission between malnutrition screening tool (NUTRIC) score and serum cytokines (IL-6) APACHE II, and SOFA scores.

Conclusion: The results of the present study revealed that pro-inflammatory cytokines (IL-6) along with other factors such as APACHE II and SOFA had significantly higher among patients with risk of malnutrition.

Keywords: pro-inflammatory cytokines, traumatic head injury (THI), nutritional score
\end{abstract}

\section{INTRODUCTION AND BACKGROUND}

Research has revealed that out of the patients admitted to intensive care unit (ICU), 14.3\% have been reported with traumatic head injuries (THI) with a mortality rate of $54 \%$ [1]. Moreover, according to the statistics reported by different research studies, malnutrition is quite prevalent, with a range between $39 \%$ and $50 \%$, among critically ill patients who are hospitalized in intensive care unit (ICU) [2, 3]. Also, it has been revealed that malnutrition in such patients can lead to poor outcomes such as high rates of hospital-acquired infections, impaired healing of wounds, prolonged hospitalization, and mortality $[4,5]$. Patients with THIs have been reported to experience numerous changes including increased energy expenditure, protein catabolism, and insulin resistance and tolerance [6-8] which can lead to systemic inflammatory response (SIRS) and ultimately release of endogenous cytokines that are effective factors in producing further metabolic changes [9]. Furthermore, as a major factor in acute-phase response, interleukin 6 (IL-6) in its increased concentrations is associated with trauma, surgery, sepsis, burn, cancer, and cardiogenic shock [10, 11].

According to the serious adverse changes caused by malnutrition in ICU patients, it is highly significant to diagnose malnutrition in critically ill patients and determine the exact time of providing nutritional support. Some researchers such as Puthucheary et al. (2013) suggested that patients in ICU should be provided with nutritional aid as soon as possible in order to minimize their metabolic response to stress caused by their conditions in ICU and to prevent subsequent oxidative cellular injury [12]. However, Heyland et al. (2011) devised a new scoring tool called Nutrition Risk in Critically ill (NUTRIC) score as the first nutrition risk assessment tool which was developed and validated specifically for patients hospitalized in intensive care units (ICUs) [13]. This tool works based on the conceptual model suggesting there is an association between the markers of acute and chronic starvation, inflammation, and outcomes [14].

NUTRIC score includes and provide measurement for different biomarkers including age, Sequential Organ Failure Assessment (SOFA) score, days from hospital admission to ICU admission, number of comorbidities, Acute Physiology and Chronic Health Evaluation (APACHE) II score, and serum interleukin 6 (IL-6) level [13]. However, Heyland et al. (2011) suggested that IL-6 can be dropped from the NUTRIC score because it only increases the c-index by 0.007 (from 0.776 to 0.783 ) which is not clinically a significant difference. This suggestion gave way to development of modified NUTRIC in which IL6 is not included in the score [15], and most studies have excluded IL-6 [16-18]. However, there is abundant 
evidence suggesting that IL-6 is strongly correlated with malnutrition inflammation in hemodialysis patients $[19,20]$, patients with lung cancer [21], patients with latent tuberculosis infection [22], and end stage renal disease patients [23]. Therefore, IL-6 can be considered as an important biomarker which cannot be easily ignored in patients hospitalized in ICU.

To the best of the author's knowledge, the relationship between NUTRIC score and IL-6 and other nutritional biomarkers has not been studied in patients with traumatic head injuries in Sulaimani, the Kurdistan Region of Iraq. Therefore, the present investigation was carried out in order to examine the relationship between malnutrition screening tool (NUTRIC) score and serum pro- and antiinflammatory cytokines levels and some nutritional parameters in THI patients in Sulaimani.

\section{PATIENTS AND METHODS}

Study population: The present investigation was a prospective cohort study. The study sample consisted of 64 patients with Glasgow comma score less than 10 who were hospitalized in the intensive care unit of General Teaching Hospital in Sulaimani, the Kurdistan Region of Iraq from 10 January, 2019 to 15 December, 2020. Patients under 18 and over 65 and those with previous chronic diseases such as diabetes, hypertension, chronic obstructive air diseases, heart diseases, malignancy, liver diseases, and history of anti-platelet medications were excluded from the study.

Assessments: NUTRIC scores was utilized to screen the patients' nutritional score, and their NUTRIC scores were derived within 24 hours from their admission, and then on days 6 and 13 of their stay in ICU. Moreover, mid-upper arm circumference (MUAC) was measured using a flexible measuring tape which was wrapped around the mid-upper arm within 24 hours from their admission, and on days 6 and 13 of their stay in ICU.

Severity of trauma: Acute Physiology and Chronic Health Evaluation (APACHE II) scoring system and Sequential Organ Failure Assessment (SOFA) score were performed to assess the severity of the disease and incidence of organ dysfunction. Both scores (APACHE II and SOFA) were obtained within 24 hours after admission, and on days 6 and 13 of their stay in ICU.

Immunoassay methods: In order to determine the concentration level of serum cytokines (IL-6), blood samples were collected from the traumatic head injured patients within 24 hours of their admission to ICU. The blood samples were placed in ice and centrifuged at 3000 $\times \mathrm{g}$, and the aliquoted plasma was frozen at $-70{ }^{\circ} \mathrm{C}$. After the blood samples were obtained from the 64 traumatic head injured patients, the immune-assay measurements were performed according to the manufacturer's instruction by using multiplexing technology as fully-automated evidence and semi-automated benchtop analyzer evidence investigator supplied by Shanghai Korain Biotech Co. Ltd. specialized in lab supplies of ELISA kit, antibodies, and protein for life science research.

Statistical analysis: After the patients' socio-demographic data including age, weight, height, and laboratory measurements and the percentage of categorical variables as gender, BMI, MUAC were collected ad expressed as mean $\pm s d$, Kolmogorov-Smirnov test was used to examine the normal distribution of the collected data. The NUTRIC scores were divided into low risk and high risk of malnutrition. Since the serum cytokines were not normally distributed, Mann-Whitney $U$ test was employed to test the significance of the collected data. In addition, the median of the cytokines and other continuous measures were compared. For normally distributed data an independent sample $\mathrm{T}$ test was used to examine the relation between NUTRIC scores and nutritional parameters (BMI). The level of statistical significance was set at $p<0.05$. Data analysis was carried out using SPSS version 25.0 (SPSS) (IBM) Inc. Chicago, IL, USA).

Ethical considerations: In order to take the ethical considerations into account in the current study, it was carried out according to the standard clinical ethics guideline, and its ethical approval was obtained from the Sulaimani university, Iraq. Moreover, after the target patients and their families were provided with explanations about the study's method and objectives, confidentiality of the data, and their freedom to quit the study, their written consent was obtained.

\section{RESULTS}

Study patient characteristics: The present cohort study was carried out on 64 patients with THI. Regarding the patients' gender, 38 were males and 26 females. Their age range was $20-64$ years with a mean of $35.97 \pm 11.5$ years. Regarding the place of their residence, most of the patients $(57 \%)$ lived in the city, and $42 \%$ resided outside the city. Moreover, most of the patients (81.2\%) were wellnourished on admission and thus at a low risk of malnutrition. The median (IQR) of their MUAC at admission were 27.2(3) .The patients' mean \pm sd of $B M I$ was $28.01 \pm 3.51$ In addition, the patients' APACHE II and SOFA scores at admission were respectively 16.17 \pm 5.07 and $11 \pm 3.57$ (See Table 1).

Table 1. The patients' socio-demographic data $(n=64)$

\begin{tabular}{|l|l|}
\hline Variables & No. $(\%)$ \\
\hline Age (Mean \pm SD)/years & $35.97 \pm 11.6$ \\
\hline Age range/years & $20-64$ \\
\hline Gender & $38(59.4)$ \\
\hline Male & $26(40.6)$ \\
\hline Female & \\
\hline Address & $37(57.8)$ \\
\hline Inside city & $27(42.2)$ \\
\hline Outside city & \\
\hline NUTRIC score at admission & $52(81.2)$ \\
\hline Low malnutrition risk & $12(18.8)$ \\
\hline High malnutrition risk & $28.01 \pm 3.51$ \\
\hline $\begin{array}{l}\text { Body mass index (BMI) } \\
\text { (Mean } \pm S D)\end{array}$ & $27.2(3)$ \\
\hline $\begin{array}{l}\text { Mid-upper arm circumference (MUAC)/cm } \\
\text { Median(IQR) }\end{array}$ & $18.0(10)$ \\
\hline $\begin{array}{l}\text { APACHE II score } \\
\text { Median(IQR) }\end{array}$ & $12(5)$ \\
\hline $\begin{array}{l}\text { SOFA score } \\
\text { Median(IQR) }\end{array}$ & \\
\hline
\end{tabular}

The data obtained within 24 hours after admission were analyzed using Mann-Whitney $U$ test and independent-samples $\mathrm{T}$ test, and the results indicated that there was a significant association between the patients' 
NUTRIC score and the studied interleukin (i.e. IL-6) $(p<0.001)$. The results also revealed that APACHE II and SOFA score had a significant relationship with the patients' NUTRIC score. Regarding, nutritional parameters, the results revealed that the patients' NUTRIC score had no significant relationship with their MUAC ( $p>0.05)$, but not with (See Table 2).

Analyzing the nutritional scores obtained 6 days after the patients were admitted to the ICU revealed that there was a significant association between the patients' NUTRIC scores and the level of their interleukin which were assessed within 24 hours after admission $(p<0.001)$. The results also showed that APACHE II and SOFA scores were significantly correlated with the patients' NUTRIC scores $(p<0.001)$. Moreover, MUAC had a significant association with the NUTRIC score $(p<0.001)$ (See Table 3).
The NUTRIC scores obtained 13 days after ICU admission, nutritional screening tools were analyzed using Mann-Whitney $U$ test and independent- sample $T$ test. The results revealed a significant relationship between the level of interleukins, measured within 24 hours after admission, and the patients' NUTRIC score $(p<0.001)$. It was also observed that APACHE II and SOFA had a significant association with the patients' NUTRIC score $(p<0.001)$. there was a significant relationship between the NUTRIC score and some nutritional parameters MUAC $(p<0.001)$ (See Table 4). The deterioration of patients condition based on NUTRIC score difference between day 13 and baseline ( First 24 hours) with interleukin 6 showed that there is highly statistical significant difference between the scoire difference and the average level of IL6 as this level is higher the prognoses ( based on NUTRIC score difference) worse.

Table 2. The association between the patients' NUTRIC score and the studied variables within 24 hours following the THI patients' admission in the ICU

\begin{tabular}{|l|l|l|l|}
\hline \multirow{2}{*}{ Measurements } & Nutritional screening tool & \\
\cline { 2 - 4 } & $\begin{array}{l}\text { Low Malnutrition risk } \\
\text { Median(IQR) } \\
\mathrm{N}=52\end{array}$ & $\begin{array}{l}\text { High Malnutrition risk } \\
\text { Median(IQR) } \\
\mathrm{N}=12\end{array}$ & P-value \\
\hline IL-6 ng/L & $90.4(56)$ & $400(110)$ & $<0.001$ \\
\hline APACHE II & $17.0(7)$ & $26.0(1)$ & $<0.001$ \\
\hline SOFA & $11.0(6)$ & $14.5(2)$ & 0.003 \\
\hline MUAC & $27.6(3)$ & $27.25(4)$ & 0.08 \\
\hline
\end{tabular}

Table 3. The association between the patients' NUTRIC score and the studied variables 6 days after the THI patients' admission in the ICU

\begin{tabular}{|l|l|l|l|}
\hline \multirow{4}{*}{ Measurements } & Nutritional screening tool & \\
\cline { 2 - 4 } & $\begin{array}{l}\text { Low Malnutrition risk } \\
\text { Median(IQR) } \\
\mathrm{N}=31\end{array}$ & $\begin{array}{l}\text { High Malnutrition risk } \\
\text { Median(IQR) } \\
\mathrm{N}=33\end{array}$ & P-value \\
\hline $\mathrm{IL}-6^{*} \mathrm{ng} / \mathrm{L}$ & $89.6(58)$ & $485.7(114)$ & $<0.001$ \\
\hline APACHE II & $12.0(4)$ & $23.0(6)$ & $<0.001$ \\
\hline SOFA & $9.0(9)$ & $16.0(4)$ & $<0.001$ \\
\hline MUAC & $27.11(3)$ & $22.8(3)$ & $<001$ \\
\hline${ }^{*}$ Cytokines were assessed within 24 hours of admission. & \\
\hline
\end{tabular}

Table 4. The association between the patients' nutritional score and the studied variables 13 days after the THI patients' admission in the ICU

\begin{tabular}{|l|l|l|l|}
\hline \multirow{2}{*}{ Measurements } & Nutritional screening tool & \\
\cline { 2 - 4 } & $\begin{array}{l}\text { Low Malnutrition risk } \\
\text { (Median) } \\
\mathrm{N}=31\end{array}$ & $\begin{array}{l}\text { High Malnutrition risk } \\
\text { (Median) } \\
\mathrm{N}=33\end{array}$ & P-value \\
\hline IL-6* ng/L & $70(45)$ & $490.8(114)$ & $<0.001$ \\
\hline APACHE II & $14.0(4)$ & $24.0(4)$ & $<0.001$ \\
\hline SOFA & $10.0(3)$ & $16.0(4)$ & $<0.001$ \\
\hline MUAC & $27.2(3)$ & $20.2(3)$ & $<0.001$ \\
\hline${ }^{*}$ Cytokines were assessed within 24 hours of admission. \\
\hline
\end{tabular}


Figure (1) the relation of the difference in Nutrec Score with interleukin 6 ( IL6)

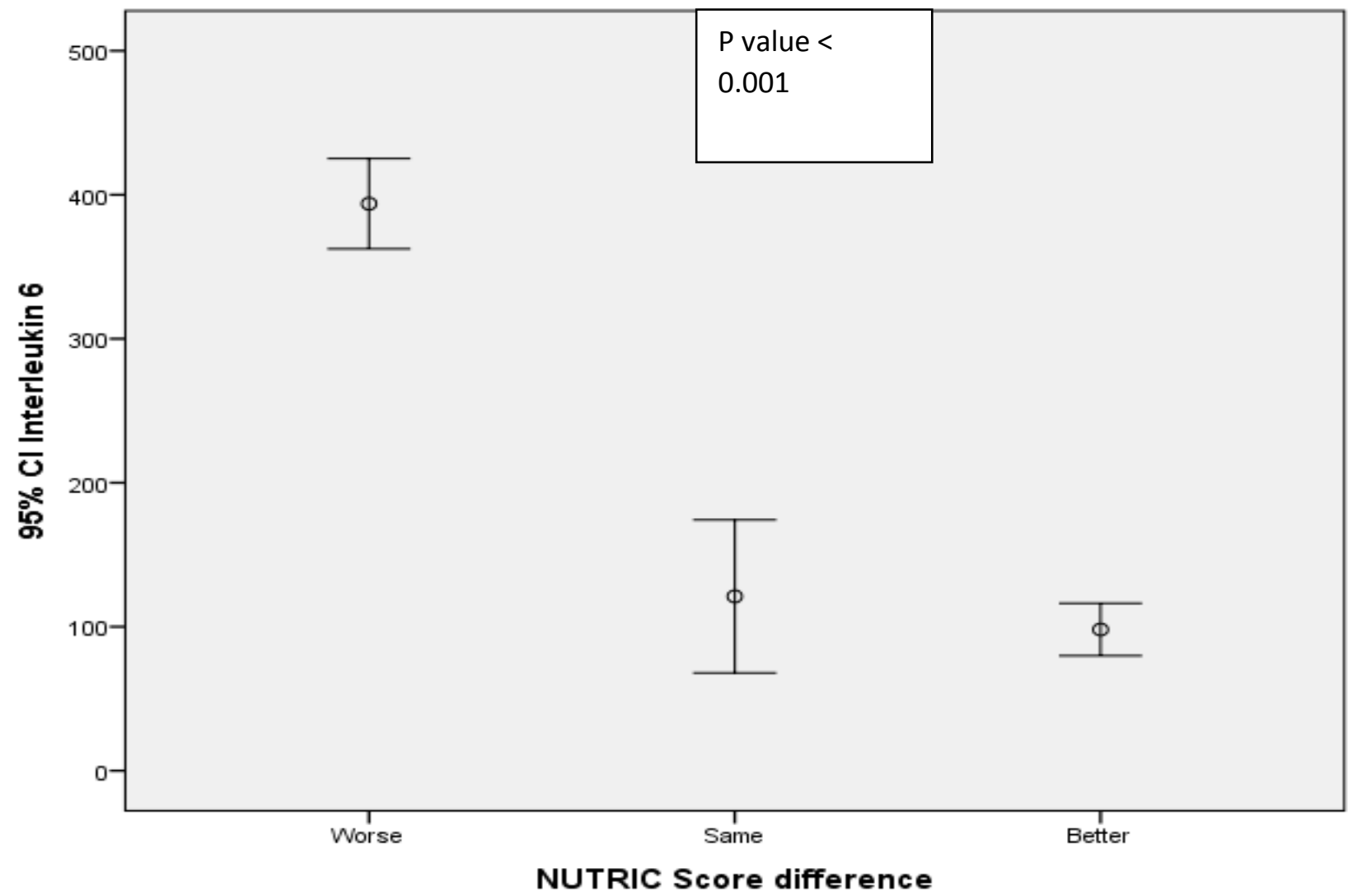

\section{DISCUSSION}

The results of the present study indicated that most of the patients $(81.2 \%)$ with traumatic head injuries were wellnourished at ICU admission time. This finding is in agreement with that of the study carried out by Chakravarty et al. (2013) who reported that $85 \%$ of the hospitalized patients with trauma were well nourished [24]. It was also observed that more than half of the patients (52\%) were at a low malnutrition risk at admission, and only $12 \%$ were at high malnutrition risk. Similar findings have been reported by Alvis-Miranda et al. (2014) and Seabury et al. (2018) $[25,26]$.

The results of the present study also indicated that there was a significant relationship between the patients' NUTRIC scores measured within 24 hours after ICU admission and the studied interleukins (i.e. IL-6) $(p<0.001)$. Santetti et al (2015) indicated a significant relationship between IL-6 and NUTRIC scores in children with chronic liver disease [27]. In line with the present study, Santetti et al (2015) [27] and Wilasco et al. (2017) [28] reported that IL-6 is significantly correlated with NUTRIC scores.

Data analysis also revealed a significant relationship between APACHE II and SOFA scores and NUTRIC scores at all stages of the study (i.e. 24 hours, 6 days, and 13 days after ICU admission). This finding is in line with those of the studies carried out by Heyland et al. (2011) [13] and Lew et al. (2017) [28]. This finding implies the correlation between APACHE II and SOFA and NUTRIC scores among critically ill patients hospitalized in ICU.
The results of the present study also showed that, there was a significant relationship between MUAC within 24 hours after ICU admission. Similar finding was reported in previously conducted studies [29, 30] however, it was observed that these measurements did not have a significant relationship with NUTRIC scores. But no similar studies were found in order to compare the findings, so more investigations need to be carried out to figure out the exact relationship between these measurements. However, it is very clear that paints whom at high risk of developing malnutrition were more prone to develop reduction in the MUAC, and BMI.

\section{CONCLUSION}

According to the results of the present study, it can be concluded that pro-inflammatory cytokines (IL-6) and other factors such as APACHE II and SOFA can be considered and utilized as reliable predictors of malnutrition risk in THI patients hospitalized in ICU. Moreover, high malnutrition risk revealed high deterioration in some nutritional parameters particularly MUAC.

\section{REFERENCES}

1. KU Tobi, AL Azeez \& SO Agbedia (2016) Outcome of traumatic brain injury in the intensive care unit: a five-year review, Southern African Journal of Anaesthesia and Analgesia 22:5,

DOI:10.1080/22201181.2016.1206293 
2. Mendes $R$, Policarpo S, Fortuna $P$, Alves $M$, Virella D, Heyland DK. (2017). Portuguese NUTRIC Study Group. Nutritional risk assessment and cultural validation of the modified NUTRIC score in critically ill patients - A multicenter prospective cohort study. J Crit Care, 37, 249.

3. Chakravarty C, Hazarika B, Goswami L, Ramasubban S. (2013). Prevalence of malnutrition in a tertiary care hospital in India. Indian J Crit Care Med, 17, 170-3.

4. de Vries, M. C., Koekkoek, W. K., Opdam, M. H., van Blokland, D., \& van Zanten, A. R. (2017). Nutritional assessment of critically ill patients: validation of the modified NUTRIC score. European journal of clinical nutrition, 72(3), 428-435.

5. Correia MI, Waitzberg DL. (2003). The impact of malnutrition on morbidity, mortality, length of hospital stay and costs evaluated through a multivariate model analysis. Clin Nutr, 22, 235-9.

6. Lee-anne SC, Chapman MJ, Lange K, Deane AM, Heyland DK. (2015). Nutrition support practices in critically ill headinjured patients: a global perspective. Critical care, 20(1), 6 .

7. Foley N, Marshall S, Pikul J, Salter K, Teasell R. (2008). Hypermetabolism following moderate to severe traumatic acute brain injury: a systematic review. Journal of neurotrauma, 25(12), 1415-31.

8. Keel M, Trentz O. (2005). Pathophysiology of polytrauma. Injury, 36(6), 691-709.

9. Jaffer, U., Wade, R. G., \& Gourlay, T. (2010). Cytokines in the systemic inflammatory response syndrome: a review. HSR proceedings in intensive care \& cardiovascular anesthesia, 2(3), 161-75.

10. Nunez Lopez, O., Cambiaso-Daniel, J., Branski, L. K., Norbury, W. B., \& Herndon, D. N. (2017). Predicting and managing sepsis in burn patients: current perspectives. Therapeutics and clinical risk management, 13, 1107-1117. doi:10.2147/TCRM.S119938

11. Gentile, L. F., Cuenca, A. G., Vanzant, E. L., Efron, P. A., McKinley, B., Moore, F., \& Moldawer, L. L. (2013). Is there value in plasma cytokine measurements in patients with severe trauma and sepsis?. Methods (San Diego, Calif.), 61(1), 3-9.

12. Puthucheary ZA, Rawal J, McPhail M, Connolly B, Ratnayake G, Chan P, et al. (2013). Acute skeletal muscle wasting in critical illness. JAMA, 310:1591-600.

13. Heyland DK, Dhaliwal R, Jiang X, Day AG. (2011). Identifying critically ill patients who benefit the most from nutrition therapy: The development and initial validation of a novel risk assessment tool. Crit Care, 15, R268.

14. Hwang SY, Lee JH, Lee YH, Hong CK, Sung AJ, Choi YC. (2012). Comparison of the Sequential Organ Failure Assessment, Acute Physiology and Chronic Health
Evaluation II scoring system, and Trauma and Injury Severity Score method for predicting the outcomes of intensive care unit trauma patients. Am J Emerg Med, 30, 749-753. doi: 10.1016/j.ajem.2011.05.022.

15. Rahman, A.; Hasan, R.M.; Agarwala, R.; Martin, C.; Day, A.G.; Heyland, D.K. (2016). Identifying critically-ill patients who will benefit most from nutritional therapy: Further validation of the "modified nutric" nutritional risk assessment tool. Clin. Nutr, 35, 158-162.

16. Jeong, D. H., Hong, S. B., Lim, C. M., Koh, Y., Seo, J., Kim, Y., Min, J. Y., Huh, J. W. (2018). Comparison of Accuracy of NUTRIC and Modified NUTRIC Scores in Predicting 28-Day Mortality in Patients with Sepsis: A Single Center Retrospective Study. Nutrients, 10(7), 911. doi:10.3390/nu10070911

17. Labarere J. (2014). How to derive and validate clinical prediction models for use in intensive care medicine. Intensive Care Med, 40, 513e27.

18. Heyland DK, Dhaliwal R, Wang M, Day AG. (2015). The prevalence of iatrogenic underfeeding in the nutritionally "at?risk" critically ill patient: results of an international, multicenter, prospective study. Clin Nutr, 34(4), 659?666.

19. Thandavan, T. ; Abraham, G., Soundararajan, P. \& Chandrasekaran, V. (2015). "Interleukin 6 Strongly Correlates with Malnutrition Inflammation Score and it is Strong Predictor for Mortality in Hemodialysis Patients". Urol. Nephrol. Open Access J, 2(2), 2-3.

20. Beberashvili, I., Sinuani, I., Azar, A., Yasur, H., Shapiro, G., Feldman, L., Averbukh, Z., ... Weissgarten, J. (2011). IL-6 levels, nutritional status, and mortality in prevalent hemodialysis patients. Clinical journal of the American Society of Nephrology : CJASN, 6(9), 2253-63.

21. Songür N, Kuru B, Kalkan F, Ozdilekcan C, Cakmak H, Hizel N. (2004). Serum interleukin-6 levels correlate with malnutrition and survival in patients with advanced non-small cell lung cancer. Tumori, 90, 196-200.

22. Anuradha R, Munisankar S, Bhootra $Y$, Kumar NP, Dolla C, Kumaran P, Babu S. (2016). Coexistent malnutrition is associated with perturbations in systemic and antigenspecific cytokine responses in latent tuberculosis infection. Clin Vaccine Immunol 23:339 -345. doi:10.1128/CVI.0000916.

23. Richa Sharma, Suraksha Agrawal, Anita Saxena, and R.K. Sharma. (2013). Association of IL-6, IL-10, and TNF-? gene polymorphism with malnutrition inflammation syndrome and survival among end stage renal disease patients. Journal of Interferon \& Cytokine Research.Jul, .ahead of printhttp://doi.org/10.1089/jir.2012.0109 